\title{
Motivación de servicio público entre funcionarios públicos chilenos*
}

\author{
Carolina Cuevas ${ }^{\dagger 1}$, Bastián González-Bustamante ${ }^{\ddagger 2} 3$, Magdalena \\ Calderón-Orellana ${ }^{\S 3} 1$, Diego Barría $\mathbb{I 4}^{4}$ \\ ${ }^{1}$ Pontificia Universidad Católica de Chile \\ ${ }^{2}$ University of Oxford \\ ${ }^{3}$ Universidad de Santiago de Chile \\ ${ }^{4}$ Universidad de Valparaíso, Chile
}

30 de enero de 2022

\begin{abstract}
Resumen
En los últimos treinta años, el campo de la administración pública en América Latina ha girado en torno a cómo introducir reformas en el sector público inspiradas por la Nueva Gestión Pública. En efecto, la mayoría de las administraciones
\end{abstract}

*Agradecemos a Hernán Frigolett, Tesorero General de la República hasta 2018, y Andrés Cerpa, Director de la Escuela de Capacitación hasta 2018, por su colaboración para desarrollar este trabajo de investigación en la Tesorería General de la República. Este artículo ha sido aceptado para ser publicado en Revista del CLAD Reforma y Democracia.

${ }^{\dagger}$ Magíster en Trabajo Social y Organizaciones, Pontificia Universidad Católica de Chile. $\boldsymbol{P}$ Av. Vicuña Mackenna 4860, Macul, Santiago, $₫$ czcuevas@uc.cl.

${ }^{\ddagger}$ Investigador Doctoral, Department of Politics and International Relations \& St Hilda’s College, University of Oxford, Reino Unido. Profesor Instructor, Departamento de Gestión y Políticas Públicas, Facultad de Administración y Economía, Universidad de Santiago de Chile (USACH), Chile. $\boldsymbol{P}$ St Hilda's College, Cowley Place, Oxford OX4 1DY, $\$ bastian.gonzalezbustamante@politics.ox.ac.uk, $\square$ bastian.gonzalez.b@usach.cl, 令 https://bgonzalezbustamante.com, ORCID iD https://orcid.org/00000003-1510-6820.

§Investigadora Doctoral, Facultad de Administración y Economía, Universidad de Santiago de Chile (USACH). Profesora Asistente Adjunta, Escuela de Trabajo Social, Pontificia Universidad Católica de Chile. $\boldsymbol{P}$ Av. Vicuña Mackenna 4860, Macul, Santiago, $\varangle$ mcaldero@uc.cl, ORCID iD https://orcid.org/0000-0003-0672-5873.

IProfesor Titular, Escuela de Administración Pública, Universidad de Valparaíso, Chile. P Gran Avenida José Miguel Carrera 4160, San Miguel, Santiago, $₫$ diego.barria@uv.cl, ORCID iD https://orcid.org/0000-0001-8906-9220. 
públicas se han enfocado casi exclusivamente en introducir este tipo de reformas, ignorando una serie de alternativas que han emergido en el debate académico. Una de esas alternativas es el enfoque de la motivación de servicio público, el cual analiza cómo ciertos factores intrínsecos, directamente asociados con la noción de servicio público, motivan el comportamiento de los empleados públicos. Este artículo tiene como objetivo fomentar un debate latinoamericano sobre cómo diseñar e implementar políticas de recursos humanos basadas en la teoría de la motivación de servicio público. A partir de una muestra no probabilística con 172 respuestas, el artículo identifica un número de factores de motivación de servicio público entre funcionarios públicos chilenos. Los hallazgos muestran que la atracción por las políticas públicas, el compromiso con el interés general y la compasión son factores tan poderosos como el amor por el dinero. Al mismo tiempo, los resultados muestran que el autosacrificio no motiva a los funcionarios públicos chilenos. A partir de estos hallazgos, se presenta una discusión sobre cómo la motivación de servicio público puede ser un enfoque útil para rediseñar políticas de recursos humanos.

Palabras clave: Motivación de Servicio Público; recursos humanos; administración pública; Chile

\section{Introducción}

En los últimos treinta años, el debate en el campo de la administración pública latinoamericana ha girado en torno a la introducción de procesos de reforma del sector público centrados en la adopción de enfoques del sector privado, especialmente en Chile (Araya y Cerpa, 2008; Morales, 2014; Ramírez-Alujas, 2004). De esta forma, se avanzó hacia la incorporación de enfoques centrados en la medición del desempeño y la gestión por resultados, lo que se materializó en el uso de la evaluación de programas, indicadores de gestión y la entrega de incentivos económicos para los funcionarios públicos, para lograr el cumplimiento de metas de gestión (véase Arenas de Mesa y Berner Herrera, 2010; Barría et al., 2013; Morales, 2014).

A pesar de que las administraciones públicas han prestado atención casi exclusivamente a iniciativas tipo Nueva Gestión Pública, en el campo académico han surgido una serie de enfoques que, centrados en el rescate de los atributos propiamente públicos de la administración pública, han discutido otras formas de gestionar las instituciones estatales. Tal es el caso de la motivación de servicio público (MSP). Desde que Perry y Wise (1990) acuñaron el concepto para destacar que los empleados públicos ven en el servicio a la comunidad una fuente de motivación, han surgido diversas líneas de 
investigación sobre el tema (Perry et al., 2010; Riba y Ballart, 2016; Wright, 2001). Si bien existen algunos estudios que plantean ciertas dudas con respecto a la validez del concepto (Bozeman y Su, 2015; Ritz et al., 2016), estas miradas son minoritarias y hoy se cuenta con una comunidad dedicada a estudiar este tema que destaca por ser cada vez más internacional, multidisciplinaria y multisectorial (Ritz et al., 2016). En efecto, los avances en el estudio de la motivación de servicio público han permitido agregar conocimiento a tal punto que hoy deben ser considerados como enfoques útiles para la gestión de las instituciones públicas (Christensen et al., 2017).

Comprender los fundamentos teóricos de la motivación permite a los gestores orientar el comportamiento de sus empleados (Fisher, 2009). Igualmente, conocer lo que motiva a los empleados públicos ayuda a los reclutadores a tomar decisiones más acertadas de contratación que permiten disminuir los costos asociados a posibles errores en aquellas contrataciones (Bright, 2008). Por esto, la gestión de la motivación debiese ser un tema central dentro de un programa de mejora de la gestión pública. Un enfoque tradicional tanto en la administración pública y privada ha sido asociar la motivación con los incentivos monetarios. En el caso chileno, la motivación de los empleados ha sido abordada, exclusivamente, a través de diversos mecanismos de asignación de incentivos económicos. La Ley 19.553, promulgada en 1998, estableció la asignación de modernización y beneficios por el cumplimiento de metas institucionales, de equipos de trabajo y por el cumplimiento de los denominados Programas de Mejoramiento de la Gestión (PMG) (Arenas de Mesa y Berner Herrera, 2010).

Es decir, la institucionalidad chilena ha abordado el tema a través de factores extrínsecos. Para hacer más complejo el uso de este instrumento para la gestión del personal, la introducción de los PMG no buscó únicamente aumentar el desempeño de los funcionarios, sino que fue resultado de las negociaciones desarrolladas en 1998 entre el Gobierno y la Asociación Nacional de Empleados Fiscales (ANEF) para mejorar las remuneraciones en el sector público (Ramírez-Alujas, 2004). Esto ha traído una serie de distorsiones, siendo la principal el hecho que los funcionarios parecen considerar este incentivo como un ingreso permanente antes que uno condicionado al desempeño. Con todo, actualmente es posible sostener que la dimensión de incentivos en el proceso de modernización no ha generado los efectos teóricos esperados (Morales, 2014). Sin embargo, ese enfoque se ve limitado por ciertas tendencias del zeitgeist, que obligan a tomar en cuenta los factores intrínsecos. Por ejemplo, la evidencia ha demostrado que hombres y mujeres tienen distintas actitudes frente al pago de bonos (Groeneveld, 2009). A ello se suma la importancia que las nuevas generaciones dan a valores como la justicia, creatividad, bienestar y diversidad (Ertas, 2015). Específicamente, en el 
campo de la administración pública, se ha documentado la importancia de los factores intrínsecos en la motivación de los funcionarios (Gould-Williams y Davies, 2005). A la vez, se ha advertido que existe una tensión entre los factores intrínsecos y extrínsecos. Sin desconocer la importancia del salario y los bonos, la evidencia en países de la OCDE reconoce que los trabajadores también se preocupan de cuestiones como la promoción el sentido de logro y la satisfacción laboral (French y Emerson, 2014; Gaki et al., 2013; Katou y Budhwar, 2012). Recientemente, a partir de evidencia del caso italiano, Ruffini et al. (2020) han demostrado que los sistemas de pago de incentivos dañan la motivación no monetaria, afectando el ethos propio de la administración pública y provocando frustración entre los funcionarios públicos. En ese sentido, pareciera ser necesario abrir un debate sobre cómo gestionar la motivación intrínseca en el sector público.

En este marco, este artículo espera contribuye a la instalación y desarrollo de políticas de gestión de personas basadas en una perspectiva de motivación de servicio público, reconociendo que las reformas fundadas en la motivación extrínseca en el caso chileno, como los incentivos monetarios, no han sido efectivas. Si bien actualmente se cuenta con información respecto a la motivación de servicio público en Latinoamérica, la región es una de las más rezagadas en estudios sobre el tema (Ritz et al., 2016). Además, su desarrollo se ha limitado a describirla sin ahondar en sus posibilidades y oportunidades para la práctica. Entre los pocos trabajos que han abordado esta cuestión, ya sea en español o analizando casos latinoamericanos, se cuenta Riba y Ballart (2016) que analizan la motivación de servicio público entre altos directivos españoles. Un segundo estudio es Sanabria-Pulido (2018), que se focaliza en el efecto de motivación de servicio público en las decisiones laborales de colombianos que formaron parte previamente de un programa de becas de postgrado en el extranjero. Por último, un tercer trabajo, que observa el efecto mediador del comportamiento cívico organizacional entre motivación de servicio público y desempeño en México, es el de Mostafa y Leon-Cazares (2016).

Este artículo busca aportar a este incipiente debate. En específico, el trabajo busca identificar cómo los factores identificados por la literatura sobre motivación de servicio público motivan a los funcionarios públicos de una agencia chilena. A partir de esta información, se promueve una discusión sobre cómo las políticas de gestión de personas pueden incorporar factores motivadores intrínsecos. De hecho, este tránsito desde la investigación hacia la práctica es uno de los principales desafíos de la motivación de servicio público (Christensen et al., 2017).

La estructura del trabajo es la siguiente. En el siguiente apartado, se debate la literatura sobre motivación de servicio público. Con posterioridad, se caracteriza la política de incentivos vigente en la administración pública chilena y la institución en la 
que se realizó el estudio. La tercera sección presenta la metodología del estudio. Luego, en el cuarto apartado, se presentan los principales resultados. Finalmente, se discuten los hallazgos y sus implicancias para la gestión de personas en la administración pública chilena.

\section{Motivación de servicio público: Tres décadas de investigación}

La literatura especializada provee tres respuestas a la pregunta por qué hay personas que quieren trabajar en el sector público. Una primera respuesta, planteada por Donahue (2008), afirma que en el sector público no existe una estructura de incentivos que haga que las personas se desempeñen mejor. Por tanto, los organismos públicos no serían capaces de atraer a profesionales competentes, quienes prefieren desempeñarse en el sector privado. De esta forma llegan al sector público aquellos trabajadores que, por sus capacidades (o ausencia de ellas), no pudieron resistir la competencia y el dinamismo del sector privado, encontrando en el ámbito público un "puerto seguro", en el cual son capaces, gracias a su estabilidad, de obtener los ingresos necesarios para tener un estilo de vida de clase media (Davis, 2010; Getha-Taylor, 2019).

Existe una segunda teoría que plantea que las personas que trabajan en el sector público eligen desempeñarse en él como parte de proceso racional de toma de decisión, ya que ahí se trabaja una menor cantidad de horas en comparación con el mundo privado, permitiendo disfrutar de un mayor tiempo libre y enfrentar un menor grado de conflicto entre el trabajo y la familia (Buelens y van den Broeck, 2007). En esta línea, con base en datos de 3.314 funcionarios del sector privado y 409 del sector público belga, Buelens y van den Broeck (2007) comprueban parcialmente que los empleados del sector público están menos motivados por recompensas monetarias. Además, este estudio documenta que los funcionarios públicos están más motivados cuando se desenvuelven en un ambiente laboral donde se les presta mayor apoyo. También resulta relevante el nivel jerárquico en la motivación y, además, se evidencia que los trabajadores públicos tienen menos conflictos entre su trabajo y familia en general. Estos últimos hallazgos

sugieren que, efectivamente, podría existir una inclinación para elegir el sector público y así tener una vida privada con menos conflictos entre el trabajo y la familia.

La tercera teoría es la motivación de servicio público, la que cuenta con alta aceptación y desarrollo en la comunidad académica en las últimas décadas (Perry et al., 2010, véase también Andersen et al., 2021). El argumento central de este enfoque afirma que 
los trabajadores en el sector público están más motivados por factores intrínsecos, a diferencia de quienes trabajan en el sector privado. La primera definición de motivación de servicio público fue propuesta por Perry y Wise (1990), quienes afirmaron que los individuos están dispuestos a responder a características únicas de las instituciones públicas. De acuerdo con la definición original, es posible distinguir tres tipos de motivos: (a) racionales, es decir, cuando los individuos están interesados en determinados servicios públicos porque se identifican con sus objetivos; (b) normativos, cuando el individuo cree en el interés público; y (c) afectivos, caracterizados por el altruismo y el deseo de ayudar a terceros (Riba y Ballart, 2016).

Otros autores, como Brewer y Selden (1998) o Bellé (2013), definen la motivación de servicio público como una fuerza motivacional que lleva a los individuos a desempeñarse con sentido público, comunidad y vocación de servicio social. Vandenabeele (2007), por otra parte, define la motivación de servicio público como un conjunto de creencias y valores que superan el interés personal e inducen conductas específicas. En resumen, existen diversas definiciones a partir de la definición base de Perry y Wise (1990), que se han centrado de forma diversa tanto en los individuos como en los valores, pero mantienen el sentido público en el centro de la definición (Brewer y Selden, 1998; Rainey y Steinbauer, 1999; Riba y Ballart, 2016; Vandenabeele, 2007).

Sin embargo, el concepto no ha estado exento de críticas. Bozeman y Su (2015) plantean que la literatura ha sido incapaz de clarificar si la motivación de servicio público es propia del sector público o si, por el contrario, también existe en el sector privado y las organizaciones sin fines de lucro. Incluso señalan que los trabajos existentes no han explicado qué es lo público en la motivación de servicio público, ni han dado señales sobre qué distingue a este constructo respecto a la motivación de servicio y el altruismo. Esto último ha sido relativizado pues Piatak y Holt (2020) han demostrado que, aunque la motivación de servicio público y el altruismo son conceptos que se superponen, el primero es un mejor predictor de comportamientos prosociales y es prometedor para explicar el deseo de participar en trabajos voluntarios (Meyer-Sahling et al., 2019).

A pesar de las críticas, este es un tema de investigación con un desarrollo creciente durante los últimos años. Efectivamente, Ritz et al. (2016) realizaron una revisión sistemática sobre 323 publicaciones enfocadas en motivación de servicio público, identificando un gran aumento en la última década y diversos patrones como los tipos de muestra, métodos usados, países estudiados, entre otros. Del mismo modo, Christensen et al. (2017) realizaron una revisión bibliográfica para confirmar el creciente aumento de las publicaciones, estableciendo aportes y lecciones para su práctica.

Además, es posible revisar al menos tres metaanálisis recientes sobre motivación de 
servicio público, uno enfocado en su relación con la satisfacción laboral (Homberg et al., 2015), otro en su relación con el desempeño (Warren y Chen, 2013) y un tercero que establece que edad y género son factores que inciden en motivación de servicio público, al igual que las diferencias culturales del contexto (Parola et al., 2019).

Retomando la definición fundacional de Perry y Wise (1990), la motivación de servicio público puede entenderse como el servicio a los demás, el sacrificio personal, y el compromiso con el interés público. Perry (1996) avanzó en determinar cuáles son los rasgos característicos de esa motivación, logrando identificar el interés por las políticas públicas, el compromiso con el interés público, la compasión y el autosacrificio. En esa línea, la motivación de servicio público cuenta con una fuerte ética del servicio que motiva a los funcionarios a desear un cambio en la sociedad y actuar para conseguirlo (Kim, 2009).

El enfoque de la motivación de servicio público plantea que las organizaciones públicas que cuentan con funcionarios motivados por servir tendrán un mejor desempeño, lo que tiene como consecuencia que estas organizaciones sean menos dependientes de recompensas extrínsecas, como las económicas (Kim, 2009) ${ }^{1}$ La motivación de servicio público ha sido utilizada como una herramienta conceptual para entender las decisiones de los trabajadores respecto al sector en el cual se desempeñan. Por ejemplo, la motivación de los empleados públicos obedece a una compleja combinación de factores relacionados con características intrínsecas o internas del trabajo y elementos extrínsecos o externos (Rainey, 2009; Riba y Ballart, 2016). A pesar de lo anterior, existe abundante evidencia respecto a que los funcionarios públicos prefieren recompensas intrínsecas antes que retribuciones monetarias, siendo quizás la más recurrente la idea de contribución al bienestar social (Perry y Hondeghem, 2008). El trabajo de Buelens y van den Broeck (2007), mencionado previamente, aporta evidencia en este sentido.

Existen trabajos que buscan responder si el desempeño aumenta gracias a la motivación (Brewer, 2008, 2010; Lewis y Frank, 2002; Warren y Chen, 2013). El artículo de Wright (2007), por ejemplo, analiza la motivación de los trabajadores públicos y el rol que juegan las misiones organizacionales. El estudio se basa en la teoría de las metas, la que busca entender cómo la misión organizacional y la motivación de servicio público afectan a los empleados. Si bien la literatura plantea que las misiones organizacionales son un factor que conduce a los individuos a elegir trabajar en el servicio público, no hay evidencia empírica que lo compruebe. La teoría de las metas afirma que la misión organizacional motiva a los empleados para cumplir con los objetivos asignados,

\footnotetext{
${ }^{1}$ Es relevante indicar que estas relaciones y mecanismos causales no han sido del todo estudiados (Bellé, 2013).
} 
ya que estos dan importancia y sentido al trabajo, además la motivación aumenta si los objetivos son alcanzables. Wright (2007) utiliza análisis multivariado para analizar 807 directivos y profesionales de una agencia pública de Nueva York. Sus principales hallazgos apuntan a que cuando los empleados entienden y valoran la misión organizacional, su motivación es mayor, de igual forma, la motivación se ve influenciada porque los empleados entiendan su trabajo y se enfrenten a metas cuya consecución es posible. Esta idea es refrendada por el estudio conducido por Desmidt (2016) quien confirmó, a través de un estudio de 1.418 empleados de una organización pública belga, que las organizaciones públicas que comunican sus misiones con mensajes de alta calidad puede aumentar la motivación intrínseca de los empleados.

Por otra parte, Bright (2008) explora si la motivación de servicio público, la satisfacción laboral, y la intención de cambiar de trabajo de los empleados públicos, son mediadas por la relación entre las personas y las organizaciones en las que trabajan (Homberg et al., 2015; Naff y Crum, 1999; Norris, 2003; Pandey y Stazyk, 2008). Bright (2008) utiliza el concepto person-organization fit, el cual se refiere a la aceptación de los valores, cultura y sentido de pertenencia por los trabajadores. Este enfoque sugiere que el beneficio de la motivación de servicio público depende del grado de ajuste entre individuo y organización. Si el ajuste es fuerte, los empleados conectan sus intereses altruistas con los objetivos de la organización y, como consecuencia, creen que esta les otorga oportunidades significativas para cumplir con su motivación personal, de esta forma además tienen una mayor satisfacción con el trabajo. Es esperable que la motivación de servicio público afecte positivamente la satisfacción laboral y negativamente las intenciones de rotación, de esta forma los individuos altamente motivados debiesen seguir así a lo largo del tiempo, aunque Moynihan y Pandey (2007) indican que puede existir una relación inversa entre la motivación y la antigüedad en las organizaciones.

En su estudio Bright (2008) busca avanzar en un entendimiento de la motivación de servicio público considerando los rasgos organizaciones presentados en el personorganization fit. Para esto realizó una encuesta a 205 empleados de agencias públicas de salud, el gobierno de la ciudad y jurisdicciones de condados locales de Indiana, Kentucky y Oregon. Se escogieron estas organizaciones por representar una muestra diversa que da cuenta de un amplio rango de ocupaciones gubernamentales y diversas localidades. Un hallazgo importante de este estudio es que la congruencia entre las personas y las organizaciones en las que trabajan emerge como un factor relevante en las actitudes y comportamiento de los empleados públicos. Este trabajo indica que la motivación de servicio público no es capaz de asegurar la congruencia entre individuo y organización, lo que implica que en ambientes organizaciones hostiles, la motivación 
puede ser de corto plazo y llevar a que los trabajadores decidan retirarse, situación que se daría con menor intensidad si los individuos tienen congruencia con las instituciones en las cuales trabajan.

Si bien la evidencia sugiere que quienes trabajan en el sector público son menos materialistas que las personas que trabajan en el mundo privado, existe un debate sobre si los factores extrínsecos han desplazado a los intrínsecos como determinantes para la toma de decisiones sobre el sector en el cual un individuo desea trabajar. Georgellis et al. (2011) analizan este asunto con un estudio longitudinal a partir de diversas bases de datos británicas que abarcan un período de 40 años. La evidencia muestra que las decisiones de movimiento desde el sector privado al público se fundan principalmente en motivaciones basadas en factores intrínsecos. Los factores extrínsecos no juegan un rol preponderante en dicha decisión, lo cual resulta interesante si se considera que los autores destacan que el sector público ofrece condiciones económicas y de derechos laborales mejores que el mundo privado. Georgellis et al. (2011) sugieren que las personas que deciden trabajar en el sector público lo hacen porque sienten satisfacción por la naturaleza del trabajo.

Por último, existen algunos estudios que muestran una relación positiva entre la motivación de servicio público y el desempeño de los funcionarios en las organizaciones. Bellé (2013), por ejemplo, presenta un estudio sobre el impacto de la motivación pública en el desempeño. Trabaja principalmente con el contacto de los empleados con los beneficiarios y las intervenciones de auto-persuasión como variables. La elección de aquellos factores se sustenta en estudios que han demostrado el efecto positivo del contacto con los usuarios en el desempeño de los trabajadores públicos. Bellé (2013) desarrolló un diseño experimental con base en una muestra de 90 enfermeras de un hospital público de Italia. Ellas fueron divididas en tres grupos de 30, uno se utilizó como grupo de control, otro grupo tuvo contacto con beneficiarios, y el último desarrolló intervenciones de auto-persuasión, las cuales tienen como fin dejar en evidencia la importancia del trabajo que se realiza. Los resultados muestran que los empleados con motivación de servicio público aumentan su rendimiento si tienen experiencias de contacto con los beneficiarios de su trabajo y que las intervenciones de auto-persuasión generan un efecto similar.

La relación entre motivación de servicio público y desempeño también ha sido reafirmada por otros estudios como el de Miao et al. (2019) quienes analizaron en China los mecanismos a través de los cuales la motivación de servicio público incidía en el desempeño. Confirmaron el efecto moderador de la identificación organizacional entre motivación de servicio público y altos niveles de desempeño. Asimismo, es posible men- 
cionar el estudio desarrollado por Stefurak et al. (2020) quienes probaron en el sector público de Alemania que las dimensiones de motivación de servicio público que se relacionan de manera directa con los resultados, son específicamente el compromiso con el interés público y la compasión, las cuales explicarían de mejor manera los efectos de la motivación de servicio público en el desempeño.

Quizás uno de los principales desafíos de la literatura sobre motivación de servicio público es pasar desde los estudios a la práctica. En los últimos años, gracias al aumento explosivo de investigaciones sobre el tema, se cuenta con un conjunto de trabajos que han permitido documentar, en países de la OCDE, la utilidad de la MSP como un complemento de las prácticas tradicionales en materia de recursos humanos (Sultana et al., 2020). Homberg et al. (2015) muestran que la motivación de servicio público es un buen instrumento para aumentar la satisfacción laboral. Además, detectaron que las dimensiones que más ayudan son la compasión y el autosacrificio, junto con la existencia de trabajos que permitan servir a la comunidad diariamente. Esto sería un buen indicador al momento de diseñar funciones. Giauque et al. (2013) y AnderfuhrenBiget et al. (2010) mostraron, a partir de un estudio en Suiza, que ciertas prácticas, como el fomento de la equidad, el enriquecimiento de cargos, la introducción de prácticas de participación y justicia, la evaluación y el desarrollo pueden aumentar los niveles de MSP. Un estudio centrado en hospitales públicos alemanes, en tanto, muestra que la capacitación, el trabajo en equipo autónomo y las reuniones fomentan la MSP, no así intervenciones en materia de comunicación, bonos de desempeño y el proceso de selección (Schott y Pronk, 2014). La capacitación también fue identificada como un motivador no monetario en el caso de funcionarios municipales en Alemania (Thaler et al., 2017). En tanto, un estudio llevado a cabo en un gobierno local en Reino Unido confirmó que el entrenamiento posee un efecto sobre la MSP (Gould-Williams, 2007).

Esta evidencia ha permitido la elaboración de orientaciones sobre cómo incorporar la MSP en la gestión pública. Ritz et al. (2016) identifican tres tipos de recomendaciones presentes en la literatura. El primer tipo apunta a medir los niveles de motivación de servicio público al momento de tomar decisiones de selección de personal. En segundo lugar, hay recomendaciones que apuntan a generar ciertas prácticas que permitan aumentar los niveles de motivación de servicio público. Finalmente, un conjunto de recomendaciones se basa en la idea de diseñar sistemas de recompensas no monetarios.

Por otra parte, Christensen et al. (2017) identifican cinco tipos de recomendaciones. $\mathrm{Al}$ igual que en el caso de Ritz et al. (2016), apuntan a utilizar la motivación de servicio público como una herramienta para seleccionar personal y la generación de ambientes de trabajo que mantengan la motivación y así aumentar el desempeño. A esto agregan 
facilitar el contacto entre trabajadores y beneficiaros para transmitir la importancia del trabajo desarrollado, dar oportunidades a los nuevos empleados de aprender los valores de servicio público y contar con líderes capaces moldear y comunicar valores de servicio público.

Si bien algunas de estas recomendaciones han mostrado ser útiles, por ejemplo, el contacto con beneficiarios (Bellé, 2013), gestionar la motivación de servicio público

no es simple. Un reciente estudio para el caso taiwanés demostró que la capacitación podría no ser eficaz para aumentar los niveles de motivación de servicio público, aunque esto puede deberse a características culturales propias de Taiwán (Chen et al., 2019). En resumen, la motivación de servicio público parece ser una guía útil para mejorar el desempeño del sector público, su implementación enfrenta desafíos de orden legal y político (Ritz et al., 2016).

\section{Antecedentes del caso}

\subsection{Modernización de la gestión pública y políticas de personal en el caso chileno}

Desde la década de 1990, Chile avanzó en un proceso de modernización de la gestión pública. Usando como marco de referencia las ideas de la Nueva Gestión Pública (Araya y Cerpa, 2008), tempranamente se implementaron iniciativas como el Plan Piloto (1993), el Programa de Mejoramiento de la Gestión y los Convenios de Desempeño Colectivo (1998), todas bajo una lógica de gestión por resultados (véase Arenas de Mesa y Berner Herrera, 2010; Barría et al., 2013; Dussauge, 2012; Ramírez-Alujas, 2004). El Plan Piloto fue relevante en la incorporación de indicadores de gestión, primero en un número reducido de servicios públicos, y fue el punto de partida para la masificación de este instrumento, que en la actualidad es utilizado transversalmente en la administración pública chilena (Barría et al., 2013). En tanto, el Programa de Mejoramiento de la Gestión y los Convenios de Desempeño Colectivo sirvieron para identificar metas en áreas de gestión y en equipos de trabajos dentro de los servicios públicos chilenos. Estos dos instrumentos, fueron incorporados en la Ley 19.553 de 1998. Esta ley fue el resultado de un acuerdo entre el gobierno y la ANEF. Los funcionarios públicos buscaban mejorar sus ingresos y el gobierno accedió a cambio de un sistema que asociara ese aumento con criterios de desempeño. De esta forma, con el Programa de Mejoramiento de la Gestión y el Convenio de Desempeño Colectivo se incorporó un esquema de pago de incentivos monetarios a los funcionarios de instituciones del gobierno central que 
cumpliesen con metas definidas a nivel institucional y de equipos de trabajo específicos dentro de las organizaciones.

Otra reforma relevante en la administración pública chilena ha sido la creación de la Dirección Nacional del Servicio Civil (DNSC) en 2003. La DNSC es un servicio público que cumple dos roles centrales. Por una parte, maneja el Sistema de Alta Dirección Pública (SADP), el cual selecciona a partir de mecanismos técnicos a los altos directivos públicos (González-Bustamante, 2020; González-Bustamante et al., 2020). Por otro lado, la DNSC se encarga del desarrollo de personas. En un inicio, tuvo la función de asesorar a las instituciones públicas en políticas de gestión de personal. En la actualidad, el servicio tiene funciones rectoras y coordina esas políticas. En 2015 la DNSC instruyó a los servicios públicos a actualizar sus políticas de gestión de personas. Dos años después, las instituciones públicas chilenas cumplieron con el mandato de dictar sus propios códigos de ética institucional (Calderón-Orellana y Araya, 2019).

En términos de gestión de la motivación el pago de incentivos económicos es el único mecanismo de motivación que existe a nivel sistémico. Los factores intrínsecos, al contrario, no han sido incorporados. Aunque la DNSC (2015) señala que las nuevas políticas de los servicios deben desarrollar acciones para generar ambientes laborales que con base en el respeto y el buen trato favorezcan el compromiso, la motivación, el desarrollo y el desempeño de las personas, no existen orientaciones sobre cómo utilizar la motivación para mejorar el rendimiento y la satisfacción laboral de los funcionarios públicos. A su vez, los incentivos económicos se encuentran bajo cuestionamiento. Recientemente, la Dirección de Presupuestos (DIPRES) encargó un estudio a la Universidad de Chile, el cual critica el sistema, entre otras cosas, por ser ritualista y establecer metas fáciles de alcanzar (Universidad de Chile, 2016). Como resultado, es común que gran parte de los servicios públicos reciban los incentivos. Esto llegó a tal punto que el $100 \%$ de los 128 servicios públicos dentro del Programa de Mejoramiento de la Gestión obtuvo el máximo incentivo por sus logros en 2016 ( La Tercera, 22 de agosto de 2017).

Tanto la crisis del sistema de incentivos económicos, como el avance que la literatura sobre motivación de servicio público ha mostrado en términos de uso de otros factores motivadores, abren una ventana de oportunidad para explorar nuevas vías para gestionar personas en el sector público chileno. 


\subsection{La Tesorería General de la República. Personal y política de gestión de personas}

La Tesorería General de la República (TGR) es un servicio público del Ministerio de Hacienda, creado en 1927 como sustituto de la Dirección del Tesoro. La misión del servicio consiste en recaudar, invertir y distribuir los fondos del sector público con eficiencia, al servicio de la ciudadanía y contribuyendo al desarrollo del país. A la Tesorería le compete distribuir mensualmente entre los servicios públicos los ingresos que percibe. Por otra parte, también debe llevar a cabo el pago de las obligaciones fiscales a su cargo, así como la de otros servicios públicos determinados por ley. Para el 2020, la TGR cuenta con una dotación autorizada de 2.062 funcionarios quienes se desempeñan en todo Chile. El servicio tiene una presencia nacional, que se distribuye de la siguiente forma: 17 tesorerías regionales ${ }^{2}, 32$ tesorerías provinciales $^{3}, \mathrm{y}$ dos oficinas.

La remuneración que reciben los funcionarios (planta y contrata) contiene componentes fijos y variables. Estos últimos han surgido como consecuencia de la introducción de incentivos asociados al cumplimiento de metas de gestión. Los principales componentes fijos de la remuneración son los siguientes: (a) haberes fijos establecidos en la Escala Única de Sueldos ${ }^{4}$; (b) bienios, asignación que se les concede a los funcionarios cuando cumplen dos años de servicio en el mismo grado; (c) asignación de zona para quienes se desempeñan en lugares con condiciones de aislamiento o altos costos de vida, esto se define por ley e implica un aumento del $40 \%$ del sueldo base; y (d) asignación por alta dirección para quienes se desempeñan en cargos directivos de segundo nivel jerárquico.

Los componentes variables de la remuneración se dividen entre aquellos que son de carácter general para gran parte de la administración pública y los específicos de la TGR. Los primeros se encuentran definitivos en el artículo $3^{\circ}$ de la Ley 19.553 que establece la asignación por modernización a través de cuatro componentes: (a) una base correspondiente al $15 \%$ de las remuneraciones; (b) un componente asociado al cumplimiento de los PMG equivalente al 3,8\% de la remuneración si el logro es mayor al $75 \%$ y menor al $90 \%$, y 7,6\% si el logro alcanza el $90 \%$ o más; (c) un aporte por el logro obtenido por equipos de trabajo o unidades respecto a sus compromisos de desempeño, equivalente a un $4 \%$ de la remuneración si el logro es mayor a $75 \%$ y menor a $90 \%$, y $8 \%$ si el logro alcanza el $90 \%$ o más; y (d) una bonificación previsional, asociada a

\footnotetext{
${ }^{2}$ La Región Metropolitana, por ejemplo, posee dos tesorerías.

${ }^{3}$ Estas se concentran principalmente entre la IV y VIII región.

${ }^{4}$ En la Escala Única de Sueldos, creada en 1974 mediante el Decreto Ley 243, se establecen grados y un sueldo base. A esto se agregan una serie de asignaciones (e.g., familiar, por responsabilidad o profesional). Estos montos son ajustados anualmente a través de la Ley de Reajuste del Sector Público.
} 
los tres puntos anteriores, cuyo rango es entre un 20,5 y un 25,62\%, dependiendo del sistema previsional del funcionario. A lo anterior se suman dos bonos más. El primero de ellos fue fijado por la Ley 19.041 para los funcionarios de los servicios del Ministerio de Hacienda ${ }^{5}$ si la recaudación neta del año anterior excede a la recaudación base del mismo período. El beneficio se paga en cuatro cuotas durante el año, y corresponde a un $24 \%$ de la base del cálculo que se forma sumando los haberes fijos y el bono de modernización. El segundo, de carácter semestral, se entrega a los funcionarios de la TGR si se cumplen las metas de recaudación de deuda morosa en cobranza. Este beneficio, que fue establecido por la Ley 19.378 actualmente varía entre un 1,6\% y un $32 \%$ con un cálculo similar al usado para el bono de modernización.

En 2014, la TGR aprobó una política de personal, la cual fue elaborada como fruto del mandato del Instructivo Presidencial 003/2013, el cual estableció que los servicios públicos deben contar con una política en la materia basada en principios como el mérito, la equidad y la transparencia. Como ha sido la tónica del sector público chileno, la motivación de los funcionarios fue abordada por la política a través de incentivos monetarios. Sin embargo, respecto a los factores intrínsecos no existe mayor desarrollo.

La TGR realiza periódicamente un estudio de clima laboral. En el estudio se hacen mediciones respecto a la motivación, la cual se centra en cuestiones como la importancia del trabajo individual para la institución, la importancia de los resultados para la unidad, la autoevaluación sobre el desempeño o el nivel de autonomía. El estudio de 2013 en particular, mencionado en la Política de Gestión de Personas como un mecanismo de retroalimentación, tuvo dos objetivos: (a) realizar un diagnóstico organizacional para evaluar la satisfacción y el ambiente organizacional de manera cuantitativa y cualitativa; y (b) detectar otros aspectos positivos y deficitarios, como también nudos críticos del ambiente y factores organizacionales para proponer alternativas de acción y recomendaciones para el desarrollo del servicio (Fuchs, 2014).

El estudio contó con una fase cuantitativa en la que se aplicó un cuestionario a 1.356 funcionarios, cubriendo el 74,4\% del universo de la institución. Dicho cuestionario contempló 88 afirmaciones agrupadas en 14 dimensiones. Para cada una de estas afirmaciones, los funcionarios debieron contestar el grado de acuerdo o desacuerdo con en una escala Likert. Una de las dimensiones consideradas fue la motivación por el trabajo entendida como poseer un trabajo interesante en el que se puedan ocupar conocimientos y habilidades. Además, se llevó a cabo un trabajo cualitativo con cinco grupos de discusión.

Las tres dimensiones mejor evaluadas por los funcionarios se asocian con la teoría de

\footnotetext{
${ }^{5}$ Con excepción de algunos servicios dependientes de la Subsecretaría de Hacienda.
} 
las metas. Los funcionarios se identifican con la institución y sus metas organizacionales y, además, tienen claridad sobre sus funciones. Además, consideran que su trabajo se importante para la unidad en la que trabajan y para la institución en su conjunto.

La TGR muestra algunos rasgos que hacen que el estudio de factores intrínsecos de motivación parezca prometedor. Por una parte, es un servicio que cuenta con un personal que se muestra identificado con la institución y su misión y, aunque los funcionarios reciben incentivos económicos de carácter general sumados a otros exclusivos del servicio como un bono por recaudación fiscal, se sienten disconformes con su trabajo, pues consideran que su desempeño no se traduce en mejoras económicas (Fuchs, 2014). Esto parece mostrar que los atractivos incentivos económicos que ofrece la TGR no logran generar satisfacción entre los trabajadores, lo que permite sugerir la posibilidad de explorar otros mecanismos de motivación.

\section{Metodología}

\subsection{Muestra}

El estudio consideró una muestra no probabilística de 600 funcionarios, a quienes se les envió un cuestionario a través de la Intranet institucional con el apoyo de la División de Personal. Esta muestra asciende al 31,5\% de los funcionarios de todo el servicio y cubrió los distintos niveles jerárquicos de la TGR. Los resultados obtenidos solo permiten realizar análisis exploratorios pues no es posible realizar inferencia estadística ya que se trabaja con un muestreo no probabilístico. La tasa de respuesta fue del $28,7 \%$ $(n=172)$.

\subsection{Medición y técnica de análisis}

La noción de motivación de servicio público introducida por Perry y Wise (1990) se puede medir empíricamente con el cuestionario original propuesto por Perry (1996). Para construir el cuestionario original se realizaron grupos focales con estudiantes de maestría de administración pública en universidades estadounidenses. Luego, se identificaron seis dimensiones teóricas asociadas a la PSM: (a) interés por las políticas públicas; (b) compromiso con el interés público; (c) deber cívico; (d) justicia social; (e) compasión; y (f) autosacrificio. Este cuestionario contó con 38 preguntas. Posteriormente, se realizó un análisis factorial confirmatorio entre estudiantes de maestría en administración pública y negocios. Esto permitió la validación de un cuestionario de 24 preguntas y la eliminación de dos dimensiones que no resultaron estadísticamente significativas: 
Tabla 1: Características de quienes contestaron la encuesta $(n=172)$

\begin{tabular}{lcc}
\hline Variables & Característica & Porcentaje \\
\hline \multirow{2}{*}{ Sexo } & Mujer & 56,40 \\
& Hombre & 43,60 \\
\hline \multirow{4}{*}{ Edad } & 24 o menos & 0,58 \\
& $25-34$ & 0,58 \\
& $35-44$ & 22,09 \\
& $45-54$ & 37,79 \\
Calidad jurídica & $55-64$ & 26,16 \\
& 65 o más & 12,79 \\
\hline \multirow{4}{*}{ Estamento } & Planta & 31,98 \\
& Contrata & 68,02 \\
\hline & Auxiliar & 9,30 \\
& Administrativo & 20,93 \\
& Técnico o experto & 20,35 \\
& Profesional & 26,16 \\
& Directivo & 23,26 \\
\hline
\end{tabular}

Fuente: Elaboración propia.

deber cívico y justicia social.

El cuestionario de Perry (1996) también ha sido validado en otros contextos diferentes al de Estados Unidos. Kim (2009, 2011) aplicó el cuestionario entre funcionarios públicos coreanos, pero redujo el número de preguntas a 14 en una primera oportunidad y a 12 finalmente. En este contexto, Kim (2009) indica que el cuestionario es útil para el contexto coreano, pero no es tan claro que la dimensión interés por las políticas públicas sea un componente válido para aquel caso. Gan et al. (2013) validaron el cuestionario entre funcionarios públicos chinos, identificando tres dimensiones: (a) atracción por las políticas públicas; (b) compasión; y (c) autosacrificio.

Al momento de realizar el trabajo de campo, el estudio de Riba y Ballart (2016) no había sido publicado, por lo cual no existía una versión en español del cuestionario. Por esto, se tradujo el cuestionario establecido por Kim (2011), el cual consta de 12 preguntas que se agrupan en cuatro dimensiones: (a) attraction to policy making (APM); (b) commitment to the public interest (CPI); (c) compassion (COM); y (d) self-sacrifice (SS). Además, se agregaron tres preguntas nuevas, ya que Liu y Tang (2011) plantean la hipótesis que el amor por el dinero (LFM) es un factor que modera la motivación de servicio público.

Cada respuesta se ha codificado en una escala de cinco puntos, donde uno significa 
Tabla 2: Dimensiones e ítems de motivación de servicio público adaptados para Chile Dimensión Ítems traducidos al español

\begin{tabular}{|c|c|c|c|}
\hline \multirow{3}{*}{ APM } & PSM1 & $Q_{1}$ & $\begin{array}{l}\text { Me interesa trabajar en programas públicos que generan } \\
\text { beneficios para el país o la comunidad a la que } \\
\text { pertenezco }\end{array}$ \\
\hline & PSM2 & $Q_{2}$ & $\begin{array}{l}\text { Me gusta compartir mis visiones sobre políticas públicas } \\
\text { con otras personas }\end{array}$ \\
\hline & PSM3 & $Q_{3}$ & $\begin{array}{l}\text { Ver a personas obtener beneficios del programa público } \\
\text { o institución en la que trabajo me entrega una gran } \\
\text { satisfacción }\end{array}$ \\
\hline \multirow{3}{*}{ CPI } & PSM4 & $Q_{4}$ & Considero que el servicio público es un deber cívico \\
\hline & PSM5 & $Q_{5}$ & $\begin{array}{l}\text { Para mí, es más importante que el servicio público } \\
\text { tenga sentido }\end{array}$ \\
\hline & PSM6 & $Q_{6}$ & $\begin{array}{l}\text { Preferiría ver a funcionarios públicos hacer lo que es } \\
\text { mejor para la comunidad en su conjunto, incluso si } \\
\text { esto afecta mis intereses }\end{array}$ \\
\hline \multirow{3}{*}{$\mathrm{COM}$} & PSM7 & $Q_{7}$ & $\begin{array}{l}\text { Me es difícil controlar mis sentimientos cuando veo } \\
\text { personas sufriendo }\end{array}$ \\
\hline & PSM8 & $Q_{8}$ & $\begin{array}{l}\text { Con frecuencia, eventos cotidianos me recuerdan cuán } \\
\text { dependientes somos unos de otros }\end{array}$ \\
\hline & PSM9 & $Q_{9}$ & $\begin{array}{l}\text { Siento solidaridad por los problemas de las personas } \\
\text { menos privilegiadas }\end{array}$ \\
\hline \multirow{3}{*}{ SS } & PSM10 & $Q_{10}$ & $\begin{array}{l}\text { Lograr un cambio en la sociedad es, para mí, más } \\
\text { importante que mis logros personales }\end{array}$ \\
\hline & PSM11 & $Q_{11}$ & $\begin{array}{l}\text { Estoy dispuesto a hacer grandes sacrificios por el bien } \\
\text { de la sociedad }\end{array}$ \\
\hline & PSM12 & $Q_{12}$ & Creo que el deber está antes que el interés personal \\
\hline \multirow{3}{*}{ LFM } & PSM13 & $Q_{13}$ & Trabajar duro para obtener dinero me motiva \\
\hline & PSM14 & $Q_{14}$ & $\begin{array}{l}\text { Los incentivos monetarios (bonos) me hacen trabajar } \\
\text { más duro }\end{array}$ \\
\hline & PSM15 & $Q_{15}$ & Estoy muy motivado por el dinero \\
\hline
\end{tabular}

Fuente: Elaboración propia a partir de Kim (2011). 
"muy en desacuerdo" y cinco "muy de acuerdo". La aplicación del cuestionario se realizó con el apoyo institucional de la TGR entre agosto y diciembre de 2014.

Los resultados fueron sometidos a un análisis factorial. Esta técnica estadística describe series de combinaciones lineales de variables, lo que permite evaluar la estructura latente de los datos. Este tipo análisis de análisis se ha popularizado durante las últimas décadas en las ciencias sociales (Afifi et al., 2012; van Belle et al., 2004). El análisis factorial descubre factores comunes que se reconstruyen linealmente de variables originales a través de la matriz de correlaciones.

El resultado de esta reconstrucción es un conjunto de coeficientes de regresión denominados cargas factoriales (factor loadings). Se utiliza una rotación ortogonal Varimax con método Kaiser (1958) que asume la existencia de varianza compartida entre los factores. La rotación genera un giro de $45^{\circ}$ contra las agujas del reloj, esto reagrupa las variables lo que arroja una matriz de componente rotados Varimax cruda (Raw Varimax), donde las cargas factoriales con comunalidades mayores tienen mayor influencia en los ejes. Se usa la normalización de Horst (1965). Para evaluar la adecuación muestra se utiliza la medida de Kaiser-Meyer-Olkin (Kaiser-Meyer-Olkin measure of sampling adequacy) (Kaiser, 1974).

\section{Resultados}

Los resultados permiten indicar que la dimensión más relevante en términos de motivación de los funcionarios públicos se conforma con PSM13, PSM14 y PSM15, precisamente las que conforman la dimensión LFM (Tabla 3). De hecho, esta dimensión explica el 33,11\% de la varianza. La alta valoración del dinero por parte de los funcionarios sugiere que la política de incentivos económicos desarrollada por el sector público chileno y la TGR es adecuada para los intereses de los funcionarios que participaron del estudio.

Los resultados también muestran la conformación de tres de las cuatro dimensiones características de la PSM: APM (PSM1, PSM2 y PSM3), CPI (PSM4, PSM5 y PSM6) y COM (PSM7, PSM8 y PSM9). La única dimensión que no se presenta claramente entre los funcionarios de TGR es autosacrificio (SS).

Otro hallazgo relevante es que estas tres dimensiones explican el 34,53\% del total de la varianza. Es decir, agrupadas superan levemente el peso del amor por el dinero. Este resultado es relevante, pues implica que la TGR cuenta con un potencial de motivación tan importante como el dinero, pero desconocido, y por tanto no gestionado por las autoridades, hasta la realización de este estudio. 
Tabla 3: Cargas factoriales y comunalidades con rotación Varimax-Kaiser para motivación de servicio público con dimensión amor por el dinero $(n=172)$

\begin{tabular}{|c|c|c|c|c|c|}
\hline Items & Factor 1 & Factor 2 & Factor 3 & Factor 4 & Comunalidad \\
\hline \multicolumn{6}{|c|}{ APM: Atracción por las políticas públicas } \\
\hline PSM1 & 0,071 & $-0,049$ & 0,797 & 0,232 & $0,696^{\star}$ \\
\hline PSM2 & 0,172 & 0,020 & 0,733 & 0,213 & $0,612^{\star}$ \\
\hline PSM3 & 0,159 & $-0,037$ & 0,862 & 0,105 & $0,781^{\star}$ \\
\hline \multicolumn{6}{|c|}{ CPI: Compromiso con el interés público } \\
\hline PSM4 & 0,181 & $-0,032$ & 0,179 & 0,819 & $0,736^{\star}$ \\
\hline PSM5 & 0,076 & $-0,098$ & 0,333 & 0,774 & $0,725^{\star}$ \\
\hline PSM6 & 0,174 & $-0,037$ & 0,137 & 0,795 & $0,682^{\star}$ \\
\hline \multicolumn{6}{|c|}{ COM: Compasión } \\
\hline PSM7 & 0,171 & 0,884 & 0,024 & $-0,007$ & $0,811^{\star}$ \\
\hline PSM8 & 0,117 & 0,877 & 0,059 & $-0,057$ & $0,789^{\star}$ \\
\hline PSM9 & 0,083 & 0,836 & $-0,108$ & $-0,049$ & $0,720^{\star}$ \\
\hline \multicolumn{6}{|c|}{ SS: Autosacrificio } \\
\hline PSM10 & 0,370 & 0,399 & $-0,006$ & 0,391 & 0,448 \\
\hline PSM11 & 0,371 & 0,200 & 0,328 & 0,341 & 0,401 \\
\hline PSM12 & 0,641 & 0,222 & 0,401 & 0,234 & $0,676^{\star}$ \\
\hline \multicolumn{6}{|c|}{ LFM: Amor por el dinero } \\
\hline PSM13 & 0,826 & 0,180 & 0,059 & 0,101 & $0,729^{\star}$ \\
\hline PSM14 & 0,735 & 0,176 & $-0,011$ & 0,346 & $0,691^{\star}$ \\
\hline PSM15 & 0,705 & $-0,034$ & 0,387 & 0,003 & $0,648^{\star}$ \\
\hline$\%$ Varianza & 33,111 & 18,324 & 8,916 & 7,286 & \\
\hline $\begin{array}{r}\dagger \text { Carga si } \\
\text { Nota: Rotación } \\
\text { Kaiser-Meye }\end{array}$ & $\begin{array}{l}\text { nificativa } \mathrm{m} \\
\text { Varimax-Ka } \\
\text {-Olkin }=0,7\end{array}$ & $\begin{array}{l}\text { distribuida } \\
\text { ser. Converg } \\
73 \text {; Esfericid } \\
\text { uente: Elabs }\end{array}$ & $\begin{array}{l}{ }^{*} \text { Contrib } \\
\text { encia de la r } \\
\text { ad de Barlet }\end{array}$ & $\begin{array}{l}\text { ición común } \\
\text { otación en la } \\
t=0,000 ; \sum\end{array}$ & $\begin{array}{l}\text { significativa } \\
\text { quinta iteración } \\
\sigma^{2}=67,637 \text {. }\end{array}$ \\
\hline
\end{tabular}

La Tabla 4 realiza el análisis factorial excluyendo las variables de amor por el dinero. Se obtienen resultados similares al análisis previo pues se distinguen todas las dimensiones de la motivación de servicio público, excepto el autosacrificio. Otro aspecto relevante es la importancia que presentan las dimensiones. La más relevante es la compasión (33,35\%). La dimensión CPI explica el 21,94\% de la varianza. Finalmente, APM es la menos relevante con un 10,50\%.

Los resultados obtenidos tras la aplicación del cuestionario muestran que la motivación de servicio público es un fenómeno que se presenta en la TGR, y en una proporción 
Tabla 4: Cargas factoriales y comunalidades con rotación Varimax-Kaiser para motivación de servicio público $(n=172)$

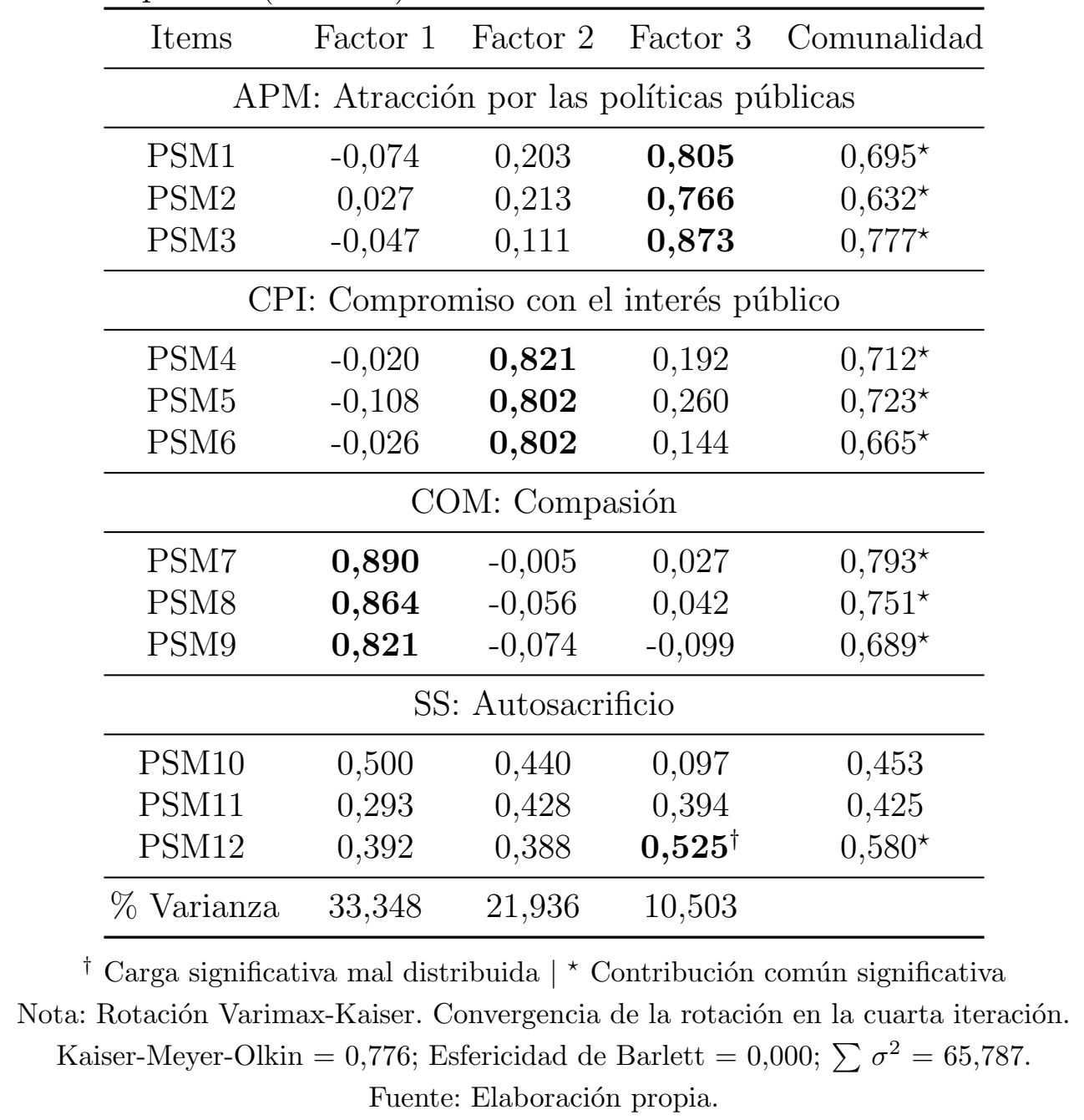

equivalente al amor por el dinero. Además, los hallazgos muestran un elemento interesante: la motivación de servicio público presente en este grupo de funcionarios chilenos solamente presenta tres de los cuatro factores considerados por el constructo. La compasión es el que tiene un mayor peso, seguido por el compromiso con el interés público y la atracción por las políticas públicas. El autosacrificio es el factor que no es significativo. Una potencial respuesta a esta ausencia puede relacionarse con la institucionalización en Chile de un sistema de incentivos económicos. En este contexto, es posible suponer que los funcionarios no están dispuestos a hacer sacrificios si aquello afecta sus ingresos. Esto sería consistente con la evidencia internacional (Liu y Tang, 2011).

De acuerdo con Homberg et al. (2015), la compasión y el interés por las políticas públicas son los factores que menos inciden en la satisfacción laboral. Al contrario, el compromiso con el interés público y la compasión son más poderosos en ese ámbito. 
Esto podría llevar a poner en duda la posibilidad de utilizar la motivación de servicio público para mejorar la satisfacción entre los funcionarios de la TGR, aunque a nivel agregado, este trabajo ha mostrado que la motivación de servicio público es equivalente al dinero. Esto abre espacio para considerar seriamente la posibilidad de incorporar esta aproximación como un factor clave en la política de personal de la institución, enfocándola como un complemento de los incentivos monetarios.

En este sentido, es relevante considerar que, por ejemplo, Frey et al. (2013) muestran que los sistemas de pago por desempeño tienen diversos problemas y efectos no deseados en el ámbito público. En esa línea, la motivación de servicio público puede ser de utilidad para resolver esos desafíos. No obstante, el desafío no es simple pues, como se ha señalado anteriormente, los incentivos económicos moderan el efecto de la motivación de servicio público.

\section{Conclusión}

La administración pública chilena cumplió recientemente dos décadas incentivando el desempeño de sus funcionarios a través del pago de incentivos monetarios. Esta modalidad ha sido ampliamente utilizada, lo que contrasta con el nulo desarrollo de iniciativas centradas en otros factores motivadores para mejorar el desempeño y la satisfacción laboral. Después de 20 años, estos incentivos se encuentran actualmente bajo una fuerte crítica. Por una parte, como muestra Fuchs (2014) para el caso de la TGR, los incentivos económicos parecen no lograr conformidad de los funcionarios con sus remuneraciones. Por otro lado, existe un fuerte cuestionamiento público y académico a la efectividad de los incentivos económicos presentes en el Programa de Mejoramiento de la Gestión y los Convenios de Desempeño Colectivo. En este contexto, resulta necesario debatir sobre la posibilidad de encontrar nuevas formas para lograr mejoras en el desempeño.

A partir de una encuesta realizada a 172 funcionarios este trabajo entrega evidencia sobre la estructura de motivación de quienes trabajan en la TGR. Los hallazgos indican, en primer lugar, que las personas se sienten motivadas principalmente por el dinero. De igual forma, a través de la aplicación del cuestionario desarrollado por Perry (1996) y validado para Corea del Sur por Kim (2009, 2011), se pudo comprobar que la motivación de servicio público es un fenómeno presente en la TGR y que su influencia es incluso levemente mayor que el del dinero.

Este hallazgo es relevante, por lo siguiente. Actualmente, el debate sobre cómo incentivar a los funcionarios públicos chilenos se ha centrado en la efectividad del Programa 
de Mejoramiento de la Gestión y los Convenios de Desempeño Colectivo para alinear incentivos económicos y resultados de gestión. En ese sentido, propuestas como la de la Universidad de Chile (2016) se centran, por una parte, en diseñar de mejor forma los instrumentos para evitar el ritualismo en la medición del desempeño institucional, junto con condicionar la entrega de incentivos económicos contra la consecución de metas más desafiantes. Los resultados que aquí se presentan cambian el foco de la discusión. En lugar de buscar un mejor diseño de la institucionalidad hoy existente, considerando que la reducción de remuneraciones de los funcionarios afectaría negativamente su compromiso, parece más adecuado ampliar el tipo de motivadores que se utilizan para lograr una mejora en el desempeño de los funcionarios públicos. Los hallazgos de este artículo muestran que las tres dimensiones de motivación de servicio público presentes en la TGR tienen un peso equivalente a los incentivos económicos. Es decir, al menos en este caso se está desaprovechando un potente factor de motivación.

Por tanto, el desafío es que en las políticas de gestión de personas de los servicios públicos chilenos la motivación deje de tener un lugar secundario y meramente enunciativo y empiece a orientar planes de acciones concretos. De todas maneras, esto no es un desafío únicamente para Chile. Como señalan Ritz et al. (2016), a pesar de la creciente evidencia internacional sobre motivación de servicio público, la academia todavía no ha sido capaz de dar respuestas sobre cómo integrar este enfoque en las prácticas de gestión de personas en el sector público. Además, como muestran Homberg et al. (2015), los servicios debiesen considerar seriamente esto, pues la satisfacción laboral aumenta cuando los trabajos dan la oportunidad a las personas de servir a la comunidad.

Los hallazgos presentados permiten sugerir que se diseñen acciones en las áreas del interés por las políticas públicas, el compromiso con el interés público y la compasión. Sobre las dos primeras, por ejemplo, la TGR podría difundir entre sus funcionarios el rol que la institución ocupa en el financiamiento de programas sociales. Igualmente, sería recomendable difundir la misión institucional entre los funcionarios, pues ella permitiría que estos entiendan la importancia de su trabajo, además de adherir a los objetivos institucionales. Por último, en relación con la compasión, una posibilidad es difundir entre el personal de la TGR historias de vida de ciudadanos que se han visto beneficiados directa o indirectamente por la función de recaudación del servicio. Esto se alinea con Bellé (2013) que destaca la importancia de la relación entre funcionarios y usuarios para mejorar el desempeño.

Finalmente, este trabajo ha permitido validar el cuestionario de motivación de servicio público en Chile. Esto abre una línea de investigación sobre los efectos de este tipo de motivación en el rendimiento organizacional y también permite reorientar los 
debates y políticas de gestión de personas en la administración pública chilena, hasta ahora hegemonizada por una mirada de Nueva Gestión Pública y por los incentivos económicos.

\section{Referencias}

Afifi, A., May, S., y Clark, V. A. (2012). Practical Multivariate Analysis. CRC Press, Boca Raton, 5th edition.

Anderfuhren-Biget, S., Varone, F., Giauque, D., y Ritz, A. (2010). Motivating Employees of the Public Sector: Does Public Service Motivation Matter? International Public Management Journal, 13(3):213-246.

Andersen, L. B., Jensen, U. T., y Kjeldsen, A. M. (2021). Public Service Motivation and Its Implications for Public Service. En Sullivan, H., Dickinson, H., y Henderson, H., editores, The Palgrave Handbook of the Public Servant. Springer International Publishing, Nueva York.

Araya, E. y Cerpa, A. (2008). La nueva gestión pública y las reformas en la administración pública chilena. Tékhne, (11):19-47.

Arenas de Mesa, A. y Berner Herrera, H. (2010). Presupuesto por Resultados y Consolidación del Sistema de Evaluación y Control de Gestión del Gobierno Central. Documento técnico, Dirección de Presupuestos del Ministerio de Hacienda de Chile, Santiago.

Barría, D., Ramírez, A., y Paris, E. (2013). Veinte años de medición del desempeño en Chile: Balance y perspectivas. Buen Gobierno, (15):44-68.

Bellé, N. (2013). Experimental Evidence on the Relationship between Public Service Motivation and Job Performance. Public Administration Review, 73(1):143-153.

Bozeman, B. y Su, X. (2015). Public Service Motivation Concepts and Theory: A Critique. Public Administration Review, 75(5):700-710.

Brewer, G. A. (2008). Employee and Organizational Performance. En Perry, J. L. y Hondeghem, A., editores, Motivation in Public Management: The Call of Public Service. Oxford University Press, Oxford. 
Brewer, G. A. (2010). Public service motivation and performance. En Walker, R. M., Boyne, G. A., y Brewer, G. A., editores, Public Management and Performance. Cambridge University Press, Cambridge.

Brewer, G. A. y Selden, S. C. (1998). Whistle Blowers in the Federal Civil Service: New Evidence of the Public Service Ethic. Journal of Public Administration Research and Theory, 8(3):413-439.

Bright, L. (2008). Does Public Service Motivation Really Make a Difference on the Job Satisfaction and Turnover Intentions of Public Employees? The American Review of Public Administration, 38(2):149-166.

Buelens, M. y van den Broeck, H. (2007). An Analysis of Differences in Work Motivation between Public and Private Sector Organizations. Public Administration Review, $67(1): 65-74$.

Calderón-Orellana, M. y Araya, R. (2019). The Codes of Ethics in the Public Sector and the Incorporation of Values that Promote Open Government. JeDEM - eJournal of eDemocracy and Open Government, 11(2):14-31.

Chen, C.-A., Hsieh, C.-W., y Chen, D.-Y. (2019). Can Training Enhance Public Employees' Public Service Motivation? A Pretest-Posttest Design. Review of Public Personnel Administration, 41(1):194-215.

Christensen, R. K., Paarlberg, L., y Perry, J. L. (2017). Public Service Motivation Research: Lessons for Practice. Public Administration Review, 77(4):529-542.

Davis, R. S. (2010). The ABCs of Public Service Motivation: Altruism, Behavior, and Compensation. Journal of Public Administration Research and Theory, 20(4):887897.

Desmidt, S. (2016). The Relevance of Mission Statements: Analysing the antecedents of perceived message quality and its relationship to employee mission engagement. Public Management Review, 18(6):894-917.

DNSC (2015). Orientaciones para el Diseño o Rediseño de Políticas de Desarrollo de Personas. Documento técnico, Dirección Nacional del Servicio Civil de Chile, Santiago.

Donahue, J. D. (2008). The Warping of Government Work. Harvard University Press, Cambridge. 
Dussauge, M. I. (2012). Transferencia de políticas y modernización administrativa. Historia (y moralejas) de cuando el Programa de Mejoramiento de la gestión chileno "viajó" a México. Revista de Gestión Pública, I(2):237-272.

Ertas, N. (2015). Turnover Intentions and Work Motivations of Millennial Employees in Federal Service. Public Personnel Management, 44(3):401-423.

Fisher, E. A. (2009). Motivation and Leadership in Social Work Management: A Review of Theories and Related Studies. Administration in Social Work, 33(4):347-367.

French, P. E. y Emerson, M. C. (2014). Assessing the Variations in Reward Preference for Local Government Employees in Terms of Position, Public Service Motivation, and Public Sector Motivation. Public Performance \& Management Review, 37(4):552-576.

Frey, B. S., Homberg, F., y Osterloh, M. (2013). Organizational Control Systems and Pay-for-Performance in the Public Service. Organization Studies, 34(7):949-972.

Fuchs (2014). Estudio de Clima Organizacional. Documento técnico, Fuchs Consultores; Tesorería General de la República de Chile, Santiago.

Gaki, E., Kontodimopoulos, N., y Niakas, D. (2013). Investigating demographic, workrelated and job satisfaction variables as predictors of motivation in Greek nurses. Journal of Nursing Management, 21(3):483-490.

Gan, K., Li, L., y Wang, Q. (2013). Public Service Motivation Measurement : A Test for Perry's Proposed Scale in China. En Proceedings of the 2013 International Conference on Public Management (ICPM-13). Atlantis Press.

Georgellis, Y., Iossa, E., y Tabvuma, V. (2011). Crowding Out Intrinsic Motivation in the Public Sector. Journal of Public Administration Research and Theory, 21(3):473493.

Getha-Taylor, H. (2019). Revitalize the Public Service, Revitalize the Middle Class. Public Administration Review, 79(5):772-776.

Giauque, D., Anderfuhren-Biget, S., y Varone, F. (2013). HRM Practices, Intrinsic Motivators, and Organizational Performance in the Public Sector. Public Personnel Management, 42(2):123-150.

González-Bustamante, B. (2020). The Politics-Administration Dichotomy: A Case Study of the Chilean Executive during the Democratic Post-Transition. Bulletin of Latin American Research, 39(5):582-597. 
González-Bustamante, B., Astete, M., y Orvenes, B. (2020). Altos directivos públicos: Un nuevo conjunto de datos de miembros del servicio civil chileno. Revista de Gestión Pública, IX(2):151-169.

Gould-Williams, J. (2007). HR practices, organizational climate and employee outcomes: evaluating social exchange relationships in local government. The International Journal of Human Resource Management, 18(9):1627-1647.

Gould-Williams, J. y Davies, F. (2005). Using social exchange theory to predict the effects of hrm practice on employee outcomes. Public Management Review, 7(1):1-24.

Groeneveld, S. (2009). Careers in the Dutch civil service: a gender perspective. International Review of Administrative Sciences, 75(3):493-507.

Homberg, F., McCarthy, D., y Tabvuma, V. (2015). A Meta-Analysis of the Relationship between Public Service Motivation and Job Satisfaction. Public Administration Review, 75(5):711-722.

Horst, P. (1965). Factor Analysis of Data Matrices. Holt; Rinehart \& Winston, Nueva York.

Kaiser, H. F. (1958). The varimax criterion for analytic rotation in factor analysis. Psychometrika, 23(3):187-200.

Kaiser, H. F. (1974). An index of factorial simplicity. Psychometrika, 39(1):31-36.

Katou, A. A. y Budhwar, P. S. (2012). The Link Between HR Practices, Psychological Contract Fulfillment, and Organizational Performance: The Case of the Greek Service Sector. Thunderbird International Business Review, 54(6):793-809.

Kim, S. (2009). Revising Perry's Measurement Scale of Public Service Motivation. The American Review of Public Administration, 39(2):149-163.

Kim, S. (2011). Testing a Revised Measure of Public Service Motivation: Reflective versus Formative Specification. Journal of Public Administration Research and Theory, 21(3):521-546.

La Tercera (2017). El $100 \%$ de servicios públicos logró bono por gestión en 2016. Nota de prensa publicada el 22 de agosto.

Lewis, G. B. y Frank, S. A. (2002). Who Wants to Work for the Government? Public Administration Review, 62(4):395-404. 
Liu, B.-C. y Tang, T. L.-P. (2011). Does the Love of Money Moderate the Relationship between Public Service Motivation and Job Satisfaction? The Case of Chinese Professionals in the Public Sector. Public Administration Review, 71(5):718-727.

Meyer-Sahling, J.-H., Mikkelsen, K. S., y Schuster, C. (2019). The Causal Effect of Public Service Motivation on Ethical Behavior in the Public Sector: Evidence from a Large-Scale Survey Experiment. Journal of Public Administration Research and Theory, 29(3):445-459.

Miao, Q., Eva, N., Newman, A., y Schwarz, G. (2019). Public service motivation and performance: The role of organizational identification. Public Money $\mathcal{E}$ Management, $39(2): 77-85$.

Morales, M. (2014). Nueva Gestión Pública en Chile: Orígenes y efectos. Revista de Ciencia Política, 34(2):417-438.

Mostafa, A. M. S. y Leon-Cazares, F. (2016). Public Service Motivation and Organizational Performance in Mexico: Testing the Mediating Effects of Organizational Citizenship Behaviors. International Journal of Public Administration, 39(1):40-48.

Moynihan, D. P. y Pandey, S. K. (2007). The Role of Organizations in Fostering Public Service Motivation. Public Administration Review, 67(1):40-53.

Naff, K. C. y Crum, J. (1999). Working for America: Does Public Service Motivation Make a Difference? Review of Public Personnel Administration, 19(4):5-16.

Norris, P. (2003). Is There Still a Public Service Ethos? Work Values, Experience, and Job Satisfaction among Government Workers. En Donahue, J. D. y Nye, J. S. J., editores, For the People, Can We Fix Public Service? Brookings Institution Press.

Pandey, S. K. y Stazyk, E. C. (2008). Antecedents and Correlates of Public Service Motivation. En Perry, J. L. y Hondeghem, A., editores, Motivation in Public Management: The Call of Public Service. Oxford University Press, Oxford.

Parola, H. R., Harari, M. B., Herst, D. E. L., y Prysmakova, P. (2019). Demographic determinants of public service motivation: a meta-analysis of PSM-age and -gender relationships. Public Management Review, 21(10):1397-1419.

Perry, J. L. (1996). Measuring Public Service Motivation: An Assessment of Construct Reliability and Validity. Journal of Public Administration Research and Theory, $6(1): 5-22$. 
Perry, J. L. y Hondeghem, A. (2008). Directions for Future Theory and Research. En Perry, J. L. y Hondeghem, A., editores, Motivation in Public Management: The Call of Public Service. Oxford University Press, Oxford.

Perry, J. L., Hondeghem, A., y Wise, L. R. (2010). Revisiting the Motivational Bases of Public Service: Twenty Years of Research and an Agenda for the Future. Public Administration Review, 70(5):681-690.

Perry, J. L. y Wise, L. R. (1990). The Motivational Bases of Public Service. Public Administration Review, 50(3):367-373.

Piatak, J. S. y Holt, S. B. (2020). Prosocial Behaviors: A Matter of Altruism or Public Service Motivation? Journal of Public Administration Research and Theory, 30(3):504-518.

Rainey, H. G. (2009). Understanding and Managing Public Organizations. Jossey-Bass, San Francisco.

Rainey, H. G. y Steinbauer, P. (1999). Galloping Elephants: Developing Elements of a Theory of Effective Government Organizations. Journal of Public Administration Research and Theory, 9(1):1-32.

Ramírez-Alujas, 1. (2004). El Proceso de Reforma del Estado y Modernización de la Gestión Pública en Chile. Lecciones, Experiencias y Aprendizajes (1990-2003). Instituto de Administración Pública, Madrid.

Riba, C. y Ballart, X. (2016). La motivación para el servicio público de los altos funcionarios españoles: medida y efectos. Revista Española de Investigaciones Sociológicas, (154):65-82.

Ritz, A., Brewer, G. A., y Neumann, O. (2016). Public Service Motivation: A Systematic Literature Review and Outlook. Public Administration Review, 76(3):414-426.

Ruffini, R., Modarelli, G., Sferrazzo, R., y Turri, M. (2020). Is merit pay changing ethos in public administration? Cogent Business \& Management, 7(1):1724703.

Sanabria-Pulido, P. (2018). Public Service Motivation and Job Sector Choice: Evidence from a Developing Country. International Journal of Public Administration, 41(13):1107-1118. 
Schott, C. y Pronk, J. L. J. (2014). Investigating and explaining organizational antecedents of PSM. Evidence-based HRM: a Global Forum for Empirical Scholarship, $2(1): 28-56$.

Stefurak, T., Morgan, R., y Johnson, R. B. (2020). The Relationship of Public Service Motivation to Job Satisfaction and Job Performance of Emergency Medical Services Professionals. Public Personnel Management, 49(4):590-616.

Sultana, S., Salman, Y., y Irfan, S. (2020). Exploring the influence of HRM Practices on Employee motivation in public organizations: A systematic Literature review. Global Management Journal for Academic \& Corporate Studies, 10(1):143-163.

Thaler, J., Spraul, K., Helmig, B., y Franzke, H. (2017). Satisfaction with and Success of Employee Training from a Public Service Motivation Perspective. International Journal of Public Administration, 40(1):1-11.

Universidad de Chile (2016). Estudio de diseño e implementación de los incentivos institucionales del sector público. Documento técnico, Universidad de Chile; Banco Interamericano de Desarrollo, Santiago.

van Belle, G., Fisher, L. D., Heagerty, P. J., y Lumley, T. (2004). Bioestatistics: A Methodology for the Health Sciences. Wiley-Interscience, Nueva York, 2nd edition.

Vandenabeele, W. (2007). Toward a public administration theory of public service motivation. Public Management Review, 9(4):545-556.

Warren, D. C. y Chen, L. T. (2013). Meta-Analysis of the Relationship between Public Service Motivation and Performance. En Ringquist, E. J., editor, Meta-Analysis for Public Management and Policy. Jossey-Bass, San Francisco.

Wright, B. E. (2001). Public-Sector Work Motivation: A Review of the Current Literature and a Revised Conceptual Model. Journal of Public Administration Research and Theory, 11(4):559-586.

Wright, B. E. (2007). Public Service and Motivation: Does Mission Matter? Public Administration Review, 67(1):54-64. 


\section{Apéndices}

\section{Biografías de los autores}

Carolina Cuevas es Magíster en Trabajo Social y Organizaciones por la Pontificia Universidad Católica de Chile y Administradora Pública por la Universidad de Chile.

Bastián González-Bustamante es doctorante en el Departamento de Ciencia Política y Relaciones Internaciones y St Hilda's College de University of Oxford, Reino Unido. Además, es Profesor Instructor del Departamento de Gestión y Políticas Públicas de la Facultad de Administración y Economía de la Universidad de Santiago de Chile (USACH). Es Magíster en Ciencia Política, Administrador Público y Licenciado en

Ciencias Políticas y Gubernamentales por la Universidad de Chile. Ha sido docente en la Universidad de Chile y consultor del Sistema de las Naciones Unidas. Ha publicado sobre gabinetes presidenciales, sistemas de servicio civil y élites políticas, gestión de crisis y gobierno electrónico en revistas como World Development, Bulletin of Latin American Research, Política \& Gobierno, Gestión \& Políticas Públicas y Convergencia.

Magdalena Calderón-Orellana es Profesora Asistente Adjunta de la Escuela de Trabajo Social de la Pontificia Universidad Católica de Chile. Actualmente está desarrollando sus estudios de Doctorado en Administración en la Universidad de Santiago de Chile (USACH). Posee un Magíster en Dirección Pública de la Universidad Católica de Valparaíso y es Asistente Social de la Pontificia Universidad Católica de Chile. En su trayectoria laboral ha combinado el ejercicio profesional, la docencia y la investigación en el área de gestión y desarrollo de recursos humanos en servicios sociales, con especial énfasis en políticas de bienestar, diversidad e inclusión en los lugares de trabajo.

Diego Barría es Doctor ( cum laude) por el Instituto de Historia de la Universiteit Leiden, Países Bajos. Magíster en Historia por la Pontificia Universidad Católica de Chile y Administrador Público por la Universidad de Chile. Es Profesor Titular de la Escuela de Administración Pública de la Universidad de Valparaíso, Chile. Ha publicado sobre historia de la administración pública, teoría de la administración pública y participación ciudadana en revistas como Bulletin of Latin American Research, Lex Localis, International Journal of Public Administration, Administrative Theory and Praxis y Latin American Perspectives. Es fundador y editor de Revista de Gestión Pública (ISSN 0719$1820)$. 


\section{Historial de revisiones}

\section{Revisión Fecha Descripción}

1,0 31 ago, 2020 Manuscrito original (MO) enviado a evaluación de pares

2,0 14 ago, 2021 MO revisado después de la primera ronda de evaluación

3,0 $14 \mathrm{sep}, 2021 \quad$ Manuscrito aceptado (MA)

$4,0 \quad 02$ nov, 2021 MA revisado

$5,0^{\star} \quad 30$ ene, $2022 \quad$ MA revisado

^ versiones disponibles en línea. 